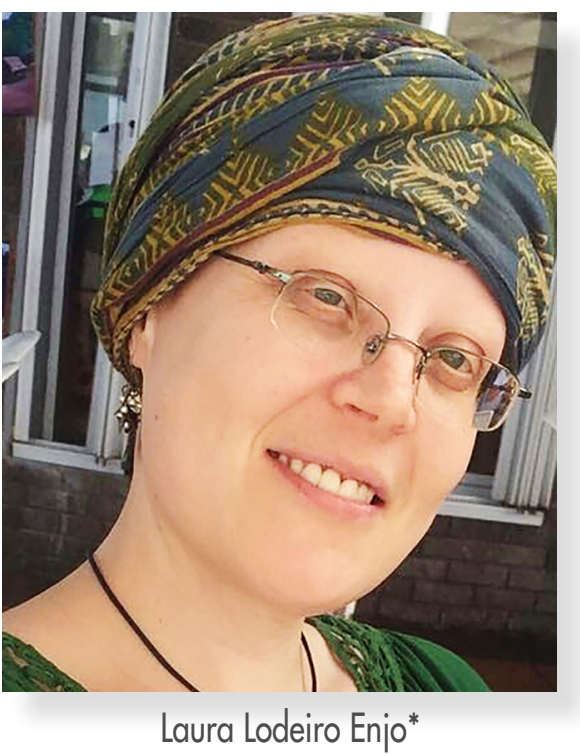

\title{
La experiencia de construcción de mapas de competencias en la Pontificia Universidad Católica Madre y Maestra (PUCMM)
}

\section{The experience of building competencies maps at Pontificia Universidad Católica Madre y Maestra} (PUCMM)

Recibido: 12-04-17 Aprobado: 08-05-17

\section{Resumen}

El Centro de Desarrollo Curricular tiene la función de ayudar y asesorar a los departamentos que así lo solicitan en el diseño de las titulaciones que ofertan en el marco del nuevo modelo educativo basado en un enfoque por competencias que ha adoptado la PUCMM. Ideamos procedimientos de diseño intrínsecamente formativos, con el propósito de ir creando condiciones facilitadoras para las siguientes etapas de desarrollo curricular. Nos integramos a las comisiones creadas en los departamentos y eso nos permite utilizar la observación participante como técnica para ir analizando la progresión de los participantes, tanto en lo que se refiere a la comprensión del nuevo modelo educativo y sus implicaciones como en el desarrollo de las competencias de trabajo en equipo y pensamiento colegiado. En este trabajo se presentan las intenciones formativas y la progresión de los participantes a lo largo de esta experiencia.

\section{Abstract}

The Center for Curriculum Development in PUCMM helps and advices the academic departments in the curriculum design process according to the University competency-based educational model. The curriculum design procedures are intrinsically formative, with the aim of creating conditions to facilitate the academic departments the following stages of curriculum development. The Center is part of the commissions for curriculum development created in the academic departments. Being part of those commissions allows us to use the participant observation technique to assess participants, both in regards to the understanding of the new educational model and its implications in the development of teamwork and collegial thought skills. This work presents the training intentions and progression of participants throughout this experience.

\section{Palabras clave}

desarrollo institucional; diseño curricular; formación del profesorado; docencia universitaria

\section{Keyword}

institutional development; curriculum design; teacher training; higher education teaching

\footnotetext{
*Laura Lodeiro Enjo: Doctora por la Universidad de Santiago de Compostela, Licenciada en Psicopedagogía y Maestra de Educación Infantil. Ha sido premio de Fin de Carrera de la Universidad de Santiago de Compostela y de la Comunidad Autónoma de Galicia en sus dos titulaciones universitarias. Es coeditora del libro Equipos Docentes y nuevas Identidades Académicas publicado en 2010. Desde enero de 2014 desarrolla su actividad profesional en la Pontificia Universidad Católica Madre y Maestra (PUCMM) como Encargada Corporativa de la Unidad de Diseño del Centro de Desarrollo Curricular (CDC) Para contactar a la autora: Ilodeiro@pucmm.edu.do
} 


\section{Introducción}

La Pontificia Universidad Católica Madre y Maestra (PUCMM) es la primera universidad privada y sin fines de lucro creada en la República Dominicana. Tiene dos campus (uno en Santiago de los Caballeros y otro en Santo Domingo) y una extensión en Puerto Plata. Actualmente vive un interesante momento de cambio que se convierte, a la vez, en un reto estimulante y una extraordinaria oportunidad desde el punto de vista del desarrollo institucional.

Se trata de un proceso de renovación integral donde la incorporación de una nueva directiva se combina con la implementación de un reciente modelo educativo, alguna reestructuración administrativa y el deseo de alcanzar una cultura académica participativa, colaborativa y abierta.

El Centro de Desarrollo Curricular (CDC) tiene la función de ayudar y asesorar a los departamentos y facultades que así lo solicitan en el diseño o rediseño de las titulaciones que ofertan. Por ello, el CDC se ha convertido en el caldo de cultivo ideal para comprender que debíamos reinventar nuestros roles y nuestro estilo formativo en función del nuevo contexto institucional en el que estamos inmersos. Necesitábamos migrar hacia una perspectiva más sistémica e integradora, degradando la imaginaria línea divisoria entre el desarrollo curricular y los procesos de formación del profesorado. La nueva perspectiva, además, debía ser multiplicadora para lograr un impacto significativo en las cuatro facultades que suman más de 20 departamentos académicos (cada uno a cargo de, al menos, una titulación de grado).

La situación que nos ha llevado a deducir esas características fundamentales que debería reunir nuestra nueva estrategia se puede resumir en las siguientes circunstancias:

- La meta de la PUCMM es contar con toda su oferta de grado en fase de implementación por competencias en un plazo máximo de 5 años y los recursos humanos del CDC para tal fin son reducidos.

- El actual Modelo Educativo de la PUCMM (PUCMM, 2011) hace referencia por primera vez a una formación por competencias y recoge una serie de competencias generales de la Universidad tomadas del Marco Pedagógico de la Universidad de Deusto en España (Universidad de Deusto, 2001). Eso supone cambiar el paradigma empleado para el diseño del curriculum de toda una universidad.
- Los planes de estudio diseñados en el marco del nuevo Modelo Educativo deben ser iguales en ambos campus en una universidad sin tradición en el trabajo colaborativo intercampus. Estamos hablando, entonces, de provocar cambios en la propia cultura institucional.

- Contamos con un profesorado poco formado y experimentado en este tipo de trabajo con sus estudiantes. Pues, aunque es de esperar que todos ellos hayan contribuido hasta el momento en el desarrollo de competencias, probablemente hayan sido pocos los que lo han hecho de una forma consciente y sistemática, con la planificación y evaluación que requiere este enfoque.

- Si bien es cierto que siempre es necesario que los docentes comprendan y actúen bajo la premisa de que un plan de estudios es un proyecto educativo en el que cada asignatura cumple con una responsabilidad concreta en aras al beneficio del conjunto; también lo es que, cuando utilizamos como unidad funcional de trabajo con los estudiantes los niveles de dominio de unas competencias, organizados cronológicamente para obtener una secuencia que vele por una progresión lógica en el aprendizaje de los estudiantes a lo largo de sus estudios de grado; resulta inevitable el divorcio entre el curriculum diseñado y el implementado si no se han creado, antes de la implementación, dinámicas y mecanismos de trabajo en equipo entre los docentes que desarrollarán un plan de estudios.

\section{Principios generales de nuestra estrategia de trabajo}

Con este marco contextual se hace evidente que, en nuestra universidad, la construcción de redes y comunidades de aprendizaje entre los docentes se convierte en un elemento clave en la mejora de la calidad de la docencia (Rué, 2007). Por otra parte, compartimos la opinión de Peter Senge, cuando afirma que los equipos (...) se están transformando en la unidad clave de aprendizaje de las organizaciones. El aprendizaje individual, en cierto nivel, es irrelevante para el aprendizaje organizativo. (...) Los individuos aprenden todo el tiempo y sin embargo no hay aprendizaje organizativo. Pero si los equipos aprenden, se transforman en un microcosmos para aprender a través de la organización. (Senge, 2005).

Además, el trabajo en equipo es la competencia más básica de las organizaciones educativas inteligentes y, al mismo tiempo, el proceso más difícil de construir 
debido a la heterogeneidad y multiplicidad de personalidades que confluyen en él (Puentes, 2006).

Parece lógico pensar, entonces, que la creación y consolidación de equipos docentes debe convertirse en una de nuestras metas logísticas prioritarias a integrar transversalmente en las iniciativas que emprendamos como Centro de Desarrollo Curricular.

Como se podrá apreciar en las explicaciones posteriores, hemos decidido seguir para ello algunas de las recomendaciones que el Dr. Roberto Di Napoli hace en referencia a las "buenas prácticas" que podrían desarrollarse de cara a la formación y gestión de equipos, así como a las condiciones que son precisas para que un modelo de trabajo en equipos docentes funcione (Di Napoli, 2010):

- Conocer al equipo, en tanto que individuos y como grupo.

- Desarrollar la narrativa del y en el grupo: favorecer que los individuos discutan los significados, las prioridades y las dinámicas del propio grupo y también de otros grupos para ver si se producen sinergias provechosas.

- Adoptar una actitud de proceso del grupo, enfocándose en las personas y no simplemente en las tareas ("task-process" vs "personprocess").

- Favorecer sensemaking, es decir la dialéctica entre las expectativas y las posibilidades reales. En el proceso se pueden encontrar soluciones creativas para el grupo.

- Promover aquellas innovaciones que apuntan a los procesos en vez de a los productos, de forma que se adquiera un verdadero conocimiento.

- Gestionar bien las diferencias de opiniones y cosmovisiones entre los individuos y los grupos. Las diferencias pueden crear una tensión creativa.

- Favorecer la creatividad y el desarrollo en vez de los aspectos "comerciales" y de gestión de cualquier innovación.

- Pensar utilizando verbos en lugar de los nombres (Ej. Introducir novedades/innovación). Se trata de movilizar, de enfatizar la acción, pasar el "tenemos que hacer" para llegar al "hacemos".
- Aceptar que hay una diferencia substancial entre el tiempo de gestión (management time) y el tiempo de aprendizaje (learning time). El segundo, al final, resulta siempre ser más constructivo que el primero que está marcado por una falta de exploración.

- Favorecer la sensibilidad del momento y la circunstancia y no siempre guiarse por el hacer pragmático.

- Equilibrar la disciplinariedad y la interdisciplinariedad.

En este sentido, desde el CDC hemos diseñado una estrategia que consiste en participar activamente en algunos equipos de los que se crean en las facultades y los departamentos académicos para desarrollar sus propios proyectos. Ellos tienen sus objetivos y nosotros ofrecemos ayuda para alcanzarlos; proponiendo y guiando procedimientos que, además, potencien la proyección a mediano y largo plazo de las iniciativas emprendidas. Es decir, nosotros velamos por mantener una visión sistémica, proyectamos en el tiempo cada facultad en función de las metas estratégicas de la Universidad y buscamos "formas de hacer" que propicien la interacción positiva entre los diferentes frentes abiertos que tienen cada una de las facultades. Perseguimos que cada proyecto o iniciativa vaya creando condiciones facilitadoras para lograr el éxito en otros venideros o que se desarrollan en simultáneo, manteniendo siempre la mirada fija en un claro horizonte institucional.

Realizamos el trabajo de formación de los docentes sin necesidad de ocuparles a los participantes un tiempo extra específico para ello y aprovechando la motivación que implica el estar recibiendo ayuda y acompañamiento para cumplir con las responsabilidades con las que deben cumplir de todos modos. Se hace un esfuerzo por facilitar aprendizajes muy significativos en un grupo reducido de profesores que se convertirá en el vértice de una pirámide de formación. Se pretende que, a través de la creación de comunidades de aprendizaje, estos sujetos formados directamente por el CDC pasen a ser tutores de sus iguales, agentes formadores que, al igual que ha hecho previamente el CDC con ellos, asesoran y acompañan a sus compañeros justo en el momento en el que deben realizar una tarea concreta. Se trata, entonces, de ir empoderando de forma paulatina a los departamentos y facultades.

Cada contacto prolongado del CDC con un grupo de profesores se convierte en una oportunidad para fortalecer en ellos competencias como el trabajo en equipo, el pensamiento reflexivo y el pensamiento 
colegiado. Al mismo tiempo, se les modela en el desarrollo de competencias generales para cuando sean ellos los que deban facilitar a sus estudiantes aprendizajes del mismo tipo.

También se les acompaña para mantenerlos activos en experiencias reales de coordinación, cooperación y/o colaboración el tiempo suficiente como para superar la etapa en la que el trabajo en equipo solamente parece dificultar y ralentizar los procesos; llegando así a otra etapa en la que las condiciones y dinámicas creadas permiten vivir las ventajas de trabajar de este modo y rentabilizar el tiempo invertido previamente (Lodeiro, 2013).

Hacerlo de este modo, implicándonos en sus proyectos, acompañándoles directamente en su desarrollo y estando presentes; también nos permite a nosotros afrontar esas iniciativas desde la perspectiva de la investigación, dotándolas del rigor y la sistematización necesarios para poder aprender de ellas y compartir nuestros aprendizajes con la comunidad científica. Así, mediante la observación participante, el registro en audio y vídeo de las sesiones de trabajo y la realización de algunas entrevistas y grupos de discusión; vamos documentando las experiencias y, a través del análisis de las evidencias, vamos aprendiendo y extrayendo conclusiones que nos permiten generar conocimiento experto. Además, serán insumos importantes para mejorar las estrategias de formación y actualización del profesorado manteniéndonos perfectamente alineados con las metas de la PUCMM.

\section{Primer procedimiento institucional diseñado desde esta perspectiva: La construcción de mapas de competencias de las titulaciones}

Las primeras iniciativas trabajando desde esta perspectiva las hemos desarrollado en el marco del diseño y desarrollo curricular de la Universidad. Específicamente con la elaboración de los mapas de competencias de las titulaciones que se ofertan en ella.

\section{Conceptos claves}

Entendemos por competencia: el conjunto de conocimientos, capacidades, habilidades o destrezas adquiridas, que da lugar a un buen nivel de desarrollo y actuación (Universidad de Deusto, 2000).

Las competencias generales de la PUCMM las hemos adoptado del Marco Pedagógico de la Universidad de Deusto y hacen referencia a aspectos vinculados con el desarrollo integral de la persona que todo profesional debe tener, independientemente de cuál sea su área de desempeño.
El conjunto de competencias específicas de una titulación es el que diferencia al profesional egresado de una carrera de los profesionales egresados de cualquier otra. Las competencias específicas son definidas por el departamento académico que gestiona la carrera a partir de la identificación de las grandes tareas sustanciales que ese profesional debe ser capaz de desarrollar y en las que moviliza aspectos conceptuales, procedimentales y actitudinales.

Tanto en un tipo de competencias como en el otro, se crea una secuencia interna de aprendizaje a través del establecimiento de niveles de dominio. Esos niveles de dominio son, en realidad, niveles de complejidad o grados de avance en la adquisición de una competencia. En nuestro Modelo Educativo se han convertido en la unidad funcional de trabajo dentro de cada plan de estudios, los adjudicados a cada asignatura indican aquello que el docente debe asegurar que va a ser exigido y evaluado a los estudiantes en la misma. Es decir, ubicar un nivel de dominio en una asignatura es asumir el compromiso de que los estudiantes que la cursen y la superen habrán demostrado un desempeño propio de dicho nivel de dominio.

Un mapa de competencias es una tabla en la que se plasma la distribución de la responsabilidad de cada una de las asignaturas con respecto al proyecto educativo completo de una titulación. Es decir, se indica en él qué niveles de dominio de competencias generales de la universidad y específicas de la titulación se alcanzarán y evaluarán en cada una de las asignaturas, así como la secuencia de aprendizaje que el estudiante seguirá a lo largo de su formación.

\section{Descripción del procedimiento y el proceso que suscita}

Para poder trabajar de este modo debíamos elegir, de entre los muchos caminos posibles para diseñar el mapa de competencias de una titulación, aquel que nos permitiese convertirlo en un procedimiento intrínsecamente formativo. Los pasos del procedimiento serían, a su vez, las actividades de un taller en el que se desarrollaría un proyecto: el propio mapa de competencias de una titulación.

Comenzamos esbozando un procedimiento basado en tres premisas fundamentales: a) el diseño curricular es vivo y dinámico, no estático; b) los documentos en los que se plasma formalmente el curriculum deben convertirse en herramientas útiles para guiar a los docentes en su día a día; y, c) del mismo modo que el curriculum formal puede incidir y modificar el curriculum implementado, éste segundo puede afectar y ocasionar la modificación del primero. 
La secuencia de experiencias de aprendizaje creada en el procedimiento busca llevar a los participantes a guiarse por las mismas premisas, a comprender las diferentes implicaciones didácticas que tienen, a utilizar competencias estructuradas en niveles de dominio y a aprovechar las ventajas que comporta. Todo ello rebajando su nivel de ansiedad ante lo nuevo y provocando actitudes positivas hacia el cambio.

Cada vez que se emprende la tarea de diseño o rediseño curricular con una titulación, se crea -en el departamento que la gestiona- una comisión intercampus delegada. Dicha comisión está conformada por un total de seis u ocho docentes que son representativos de las principales áreas implicadas en la carrera, con los directores del departamento encabezando el grupo. Una persona del CDC (siempre la misma) se une al equipo y asume, junto con ellos, la responsabilidad de sacar adelante el proyecto. El taller se desarrolla a través de reuniones presenciales de aproximadamente 6 horas con una periodicidad semanal durante seis meses.

Una de las grandes ventajas de este proceso es que la persona del CDC que trabaja con las comisiones no es una profesional de su misma área. Eso ayuda a todos a diferenciar el rol de la asesora pedagógica del de los demás integrantes de la comisión, comprendiendo que se trata de una relación interdisciplinar en la que todos sienten una responsabilidad compartida y son necesarios para sacar adelante un proyecto común alrededor del que se aúnan los esfuerzos de todos. La mayéutica se convierte, así, en la estrategia reina para el avance en el proceso, puesto que la asesora obtiene la información disciplinar para poder guiarlo a través de las respuestas de los propios participantes.

La dinámica interna de las sesiones es de seminario reflexivo, trabajando sobre un producto parcial concreto. Dicho producto suele estar plasmado en un documento digital proyectado y que se va modificando -en el momento y a la vista de todos- con las decisiones consensuadas y unánimes a las que llegan los participantes discutiendo y utilizando la argumentación para convencerse unos a otros. De este modo se van creando algunas de las condiciones necesarias para que la comisión pueda llegar a convertirse en un equipo docente, pues se genera un clima de confianza y respeto en el que se valora el aporte de cada uno.

Ninguna de las decisiones importantes en el seno de la comisión se toma de forma arbitraria o por la mayoría obtenida en una votación. "Los equipos deben aprender a explotar el potencial de muchas mentes para ser más inteligentes que una persona sola" (Senge, 2005, p. 297) y los integrantes de los mismos deben sentirse convencidos y no vencidos. Ellos serán nuestros principales motores del cambio y, por tanto, deben creer en el proyecto educativo que están construyendo y tener un convencimiento lo suficientemente fuerte y fundamentado como para ayudarnos a combatir la inevitable tendencia a la homeostasis que caracteriza a las instituciones académicas.

Utilizamos la primera experiencia con una comisión para terminar de definir el procedimiento general que seguimos. Ajustamos los diferentes pasos, su posición en la secuencia completa y la transición entre ellos en función de las situaciones reales que se nos presentaban con los participantes.

Antes de empezar a trabajar con nosotros, los integrantes de las comisiones esperan afrontar la tarea como lo han venido haciendo hasta ahora: pensando qué asignaturas incluir en el plan de estudios para luego decidir qué se trabajará en cada una de ellas. No obstante, nos hemos dado cuenta de que es más factible que los profesores comprendan las implicaciones del nuevo modelo educativo desprendiéndose primero del anterior, en el que tienen una larga trayectoria.

Por supuesto, no se pretende despreciar esa trayectoria, simplemente se posterga su aprovechamiento al momento en el que los participantes conocen lo suficiente ambos modelos como para poder compararlos y enriquecer el nuevo con lo aprendido de la experiencia en el anterior.

A continuación, se enumeran los 10 pasos que constituyen el procedimiento general que seguimos con las comisiones para la construcción del mapa de competencias de una titulación determinada.

En esa secuencia se puede apreciar que nuestra propuesta consiste en finalizar justamente en el punto en el que ellos suelen esperar comenzar:

1. Elaborar la lista inicial de las competencias específicas de la titulación.

2. Definir y establecer los niveles de dominio de las competencias específicas.

3. Socializar las competencias específicas e incorporar los ajustes derivados de la misma.

4. Analizar las relaciones temporales entre competencias específicas por niveles de dominio.

5. Establecer el periodo en el que se comenzará a trabajar cada nivel de dominio de las competencias específicas y los periodos en los que se repetirán. 
6. Analizar cuáles son los contenidos que es necesario abordar en cada periodo para cumplir con las competencias del mismo.

7. Identificar las asignaturas necesarias para desarrollar los contenidos y competencias de cada periodo.

8. Elaborar el pensum de la titulación.

9. Analizar la interacción entre las competencias generales de la Universidad y las competencias específicas de titulación.

10. Ubicar en periodos académicos los niveles de dominio de las competencias generales de la Universidad. Completar el mapa de competencias.

En cada uno de estos pasos las comisiones generan un producto parcial y, a su vez, los productos parciales, sirven para obtener un borrador sobre el que iniciar el análisis del siguiente paso. En definitiva, los pasos del procedimiento propuesto se van encadenando unos con otros de forma tal que se dotan de sentido mutuamente. A partir de las necesidades que detecta en cada momento y las características cognitivas que identifica en la comisión, la asesora pedagógica va diseñando y aportando plantillas y herramientas para que los participantes puedan comprender el tipo de análisis que deben realizar en cada momento y plasmar las decisiones que van tomando en función del mismo.

Los cambios introducidos en los productos parciales podrían entenderse como un registro de los avances tangibles de cada sesión, pero sería un gran error interpretarlos como el único insumo a partir del cual valorar la contribución que este proceso hace al desarrollo institucional de la PUCMM.

Los diferentes pasos de la secuencia se plantean con propósitos concretos y aportan valores intangibles agregados que, sin duda, redundan en beneficio de la Universidad proyectada en el mediano y el largo plazo:

Con el primero de esos pasos se pretende que los participantes, a través de la identificación de las posibles competencias específicas de su carrera, comiencen a destilar la esencia de su profesión, sus elementos constituyentes básicos.

Para hacerlo tienen que disgregar las partes integradas en su pensamiento y quehacer profesional y eso los lleva a darse cuenta de hasta qué punto comparten o no la misma visión sobre la profesión y a descubrir que les queda trabajo por hacer para llegar realmente a manejar un lenguaje común en el seno del equipo.
El segundo paso, que consiste en definir y establecer los niveles de dominio de cada competencia, sirve para que profundicen en el concepto de competencia, para que analicen las coincidencias y las diferencias en sus modos de entender la profesión, para que comiencen a conocerse desde ese punto de vista, para que se enfrenten entre ellos y para que aprendan a acercar posiciones a través de la argumentación y la construcción colegiada del pensamiento. De este modo, se van poniendo de acuerdo sobre la filosofía e intenciones estratégicas que sostienen su plan de estudios y van construyendo un lenguaje compartido, al mismo tiempo que intercambian experiencias profesionales o de aula y van diluyendo la mitificada rivalidad entre un campus y el otro.

El trabajo de la asesora en esta etapa consiste en potenciar todo lo anterior y en velar porque los docentes mantengan el foco en el aprendizaje de los estudiantes para que diseñen -con los niveles de dominioprogresiones lógicas, coherentes y realistas de sus aprendizajes, sin confundirlas con las secuencias procesuales propias de su profesión. En este paso se completa el primer borrador de las competencias específicas de la titulación.

La socialización de ese documento es un momento estratégico clave dentro del proceso: sirve para oxigenar y revitalizar la comisión, dado que sus integrantes ya llevan unos meses intercambiando opiniones y se han convencido mutuamente. Necesitan contar con el análisis crítico de otras personas que también desarrollarán el proyecto educativo en la práctica para enriquecer y validar su trabajo. Además, es la oportunidad de hacer que esos colegas se sientan informados y partícipes de la empresa en la que está embarcado su departamento deslizando el halo de misterio que rodeaba hasta ese momento a la comisión.

Intencionalmente la asesora no participa en esta sesión, pues se trata del momento elegido para que los comisionados demuestren estarse empoderando en el nuevo modelo educativo y puedan ser percibidos por sus colegas como líderes del cambio, como las personas que les explican el nuevo enfoque y responden a sus inquietudes utilizando ejemplos propios de su disciplina. Tras esa sesión la comisión realiza en las competencias específicas los ajustes derivados de los comentarios de sus colegas y hace del conocimiento de éstos la incidencia que han tenido sus opiniones y sugerencias en el producto de la comisión.

Una vez obtenido el documento final de las competencias específicas, la comisión cuenta con la materia prima para continuar avanzando. Cuando establecen las relaciones temporales entre los niveles de dominio de las diferentes competencias específicas 
en el cuarto paso, la intención es que sientan que han estado creando las piezas de un rompecabezas y que ahora empiezan a encajar. Es el momento en el que realmente comprenden porqué eran necesarias las diferentes discusiones que han mantenido con la intención de delimitar y redactar correctamente las competencias y sus respectivos niveles de dominio, pues comienzan a entender su utilidad como unidades funcionales de trabajo en el marco de un plan de estudios.

En el paso número cinco se establece la columna vertebral que sostendrá la titulación completa. A través de esta tarea definirán la progresión desde las unidades fragmentadas en el primer periodo de la titulación hacia asignaturas cada vez más integradoras y próximas a la actuación característica de un profesional. El propósito en esta ocasión es que experimenten las ventajas inherentes al establecimiento previo de unos niveles de dominio perfectamente delimitados y comprendan el significado de utilizarlos como unidades funcionales.

Los pasos seis, siete y ocho suponen la confluencia del nuevo modelo educativo y el modelo en el que los participantes han estado tradicionalmente inmersos. Es el momento que gira en torno a la idea de que las competencias deben movilizarse a través de contenidos concretos que llevan, no solamente al desarrollo de elementos procedimentales, sino también a una sólida fundamentación teórica y el desarrollo de actitudes apropiadas. También es la ocasión para incidir en la idea de que cada nivel de dominio puede alcanzarse en diferentes asignaturas o, incluso, se le podría dar un tratamiento transversal.

Los participantes suelen entender de inicio las competencias generales de la Universidad como totalmente ajenas a las titulaciones y las competencias específicas que diseñan para ellas, piensan incluso que se trabajan en asignaturas diferentes a aquellas en las que se van a desarrollar las competencias específicas de la titulación.

Contrarrestar esta visión es el objetivo de los dos últimos pasos de la secuencia. En ellos analizan hasta qué punto necesita a las diferentes competencias generales cada una de las competencias específicas de la titulación para poder ser desarrollada hasta su máximo nivel de dominio y, de este modo, los propios docentes llegan a la conclusión de que ambos tipos de competencias están íntimamente relacionadas y de cómo las competencias generales tienen mucha incidencia en aspectos metodológicos del plan de estudios. Finalmente se obtiene un mapa de competencias en el que se ven claramente relacionadas las generales y las específicas ubicadas en las diferentes asignaturas y que llegan a percibir como una herramienta fundamental para analizar la viabilidad de posibles cambios futuros en el plan de estudios.

\section{Resultados y conclusiones}

Nuestra experiencia aplicando esta estrategia todavía es corta, pero nos arroja evidencias esperanzadoras que apuntan hacia la idea de que la perspectiva de la "formación horizonte" es la adecuada para nuestro contexto actual.

Estamos hablando de algo más de dos años en los que hemos apoyado los procesos de diseño de los planes de estudios, pues todas las facultades se encuentran inmersas en una etapa de rediseño de las titulaciones que ofertan -para actualizarlas y ajustarlas a los lineamientos del nuevo modelo educativo- $y$, más adelante, tendrán que afrontar la implementación de esos proyectos educativos diseñados y convertirlos en una realidad en las aulas.

Después de culminar el diseño del mapa de competencias, los departamentos amplían las comisiones con profesores que se van incorporando para el diseño de los programas de asignaturas.

En esa nueva fase el CDC mantiene cierto liderazgo como guía mientras entrena a una persona del propio departamento para que sea la que lleve las riendas del diseño de programas hasta que el departamento termine esa labor. De este modo, además de incrementar la cantidad de agentes formadores desde el punto de vista de la pedagogía, tratamos de mitigar la circunstancia de que la enseñanza universitaria sea "uno de esos entornos humanos que raramente se beneficia de su pasado" (Bain, 2005, p. 13).

Hemos comprobado que, cuando eso sucede, el departamento ya está empoderado, no sólo puede continuar avanzando de forma autónoma en el diseño de programas de asignaturas, sino que, además, cuenta con personas que conocen en profundidad los conceptos fundamentales relacionados con las competencias, así como las implicaciones técnicas y didácticas del modelo educativo de la Universidad.

Esto implica que, completado un proceso acompañamiento del CDC, los departamentos académicos están listos para hacer cuantas modificaciones sea necesario en el marco del modelo educativo actual, sin necesidad de contar con la presencia de algún integrante de nuestro centro.

Además, y no menos importante, los departamentos se sienten capacitados para hacerlo y el CDC tranquilo al dejar esas cuestiones en sus manos. En los participantes de las comisiones hemos detectado una clara evolución en lo que respecta a sus apreciaciones sobre las potencialidades de los equipos docentes y a la necesidad sentida de trabajar desde un enfoque de construcción colegiada del pensamiento. En las primeras 
sesiones presentan una fuerte resistencia a hacerlo y, a medida que avanzan en el proceso, son cada vez más las ocasiones en las que manifiestan no poder tomar una decisión determinada porque necesitan para ello escuchar la opinión de algún integrante de la comisión ausente o de algunas personas clave no pertenecientes a la comisión.

La experiencia nos está demostrando que es necesario mantener la dinámica interna de toma de decisiones unánimes en el seno de las comisiones. Pues son muchas las ocasiones en las que nos encontramos con una única nota discordante que aporta un factor determinante a partir del cual se origina alguna característica esencial del proyecto educativo de la que los autores se sienten satisfechos y orgullosos.

Se produce una tendencia semejante en lo que se refiere a la aceptación del rol de la asesora pedagógica que se implica con la comisión. Al comienzo cuestionan el hecho de que una persona ajena a su área de especialización pueda hacer algún aporte de interés al diseño de su titulación o los programas de las asignaturas que lo componen. No obstante, después de pasadas tres o cuatro sesiones, comprenden que su papel no es el de decirles lo que tienen que aprender sus estudiantes sino el de guiarles en sus procesos reflexivos a través de un método socrático para asegurarse de que se hagan a sí mismos las preguntas que deben hacerse a la hora de idear esos planes de estudios desde el Modelo Educativo de la PUCMM.

Nos preocupa un poco la progresión ascendente de la apreciación positiva del aporte de la asesora pedagógica porque, hacia el final del acompañamiento, es tan elevada que, en ocasiones, no quieren pasar a la etapa en la que avanzan de forma autónoma, porque la figura del CDC les aporta seguridad. Aún así, nosotros pasamos a un segundo plano (a su disposición para responder cuestiones puntuales y hacer una evaluación técnica) en el momento en que estimamos que ya están listos para ello. Pero de ello deducimos que debemos incorporar algún ajuste en la dinámica para reducir la resistencia inicial a pasar a la fase de desarrollo autónomo, para que se incremente su confianza en ellos mismos.

En ocasiones nos sorprenden cambios actitudinales que se producen en algunos participantes en especial, porque superan con creces nuestras expectativas. Nos referimos a casos como el de aquellos docentes que, tras haberse mostrado de inicio totalmente escépticos con respecto al modelo educativo, se convierten meses después en sus defensores más acérrimos y en los mejores apoyos que encuentran los directores a la hora de continuar la experiencia en la etapa autónoma. Interpretamos que, en parte, eso viene dado por la dinámica dialogante que se genera en el seno de la comisión. La propia asesora también debe convencer a los demás con argumentaciones y explicaciones, al mismo tiempo que demuestra que cada tarea solicitada tiene un sentido y una utilidad dentro del proceso.

Otro caso interesante es el de un profesor que al principio no se dirigía a los demás (ni siquiera la mirada, la mantenía fija en la pantalla en la que se iban proyectando los acuerdos tomados) y que, pasados cuatro meses, incluso bromeaba con sus compañeros y propiciaba el dinamismo de las sesiones. Es un ejemplo entre los muchos que nos permiten afirmar que se están produciendo avances importantes en lo que se refiere a las relaciones interpersonales y académicas que se dan entre profesores de un mismo departamento en un mismo campus y entre profesores de un mismo departamento de diferentes campus.

En los últimos meses estamos percibiendo un interesante fenómeno que apunta en la misma línea: La persona del CDC que trabaja con las comisiones es siempre la misma y eso le permite mantener un enfoque comparativo entre las diferentes carreras para enriquecer unas comisiones con la experiencia y producciones de otras. En ocasiones, las propias comisiones generan herramientas o modos diferentes de hacer los análisis que la asesora deja fluir si no perjudican el proceso e incorpora a su banco de recursos para proporcionarles la alternativa a otros equipos en los que identifica características o estilos semejantes.

Lo interesante es que esa perspectiva parece permear también en los docentes que participan en las comisiones y ya tenían unas buenas relaciones entre ellos antes de pasar por el proceso. Nos hemos percatado de que cada vez son más frecuentes los encuentros informales o incluso casuales en los que los participantes en una comisión consultan a los participantes de otra sobre alguna cuestión de común interés o en los que participantes de un departamento avanzado explican a los de otro que está comenzando su andadura que deben tener un poco de paciencia porque pronto lo entenderán todo.

De hecho, cuando preguntamos a los integrantes de comisiones cuyos planes de estudios ya se han aprobado sobre cómo se han sentido en la experiencia, nos explican que, aunque entienden que así es como debe hacerse, se trata de un proceso prolongado y afanoso. También nos indican que al principio se sentían perdidos y que, de repente, parecía que todo encajaba como en un gran rompecabezas. Eso nos hace pensar que se están produciendo aprendizajes significativos.

Nos llaman la atención sobre el hecho de que es un trabajo que requiere de mucha dedicación y esfuerzo, motivo por el que consideran que los participantes deberían ser recompensados y reconocidos de algún modo por realizarlo. 
A partir de la mitad del proceso se percibe que comienzan a sentirse orgullosos de su trabajo, de las producciones que generan y eso les da aliento para continuar. Es también en ese momento cuando, casi la totalidad de las comisiones, comienzan a preguntarle a la persona del CDC que trabaja con ellos si esa es su comisión favorita, dando por sentado que así es. Esa pregunta suele surgir, o bien cuando logran resolver una cuestión complicada o bien en momentos distendidos de algunas reuniones. El hecho de que se sientan "los favoritos" es interpretado por nosotros como un indicativo de que se ha creado un clima agradable, de confianza y de respeto en la comisión. Ellos, en cierto modo, disfrutan el proceso y se percatan de que la persona que les acompaña también lo hace.

A través del proceso se están fortaleciendo de forma natural los flujos comunicativos entre los directores de Santiago y Santo Domingo y surgen interesantes iniciativas colaborativas entre diferentes profesores de la misma comisión, independientemente del campus al que pertenezcan.

Sin embargo, también nos hemos dado cuenta de que esos flujos comunicativos, aunque fortalecidos, todavía no lo están lo suficiente. Estamos viendo como, en algunas situaciones puntuales, hay directores que regresan a la práctica anterior en la que un campus funcionaba casi como si el otro no existiese. Esto nos lleva a pensar que tendremos que prestar especial atención a este aspecto en futuros proyectos con las facultades y los departamentos académicos, además de pensar modos de mantener activas las dinámicas creadas para que no se produzcan retrocesos en los avances.

Probablemente podría contribuir a enfatizar y mantener el sentido de unidad el incorporarlo de forma explícita a los procesos y flujos de comunicación interna de la Universidad. Nos referimos a cuestiones como, por ejemplo, sugerir a los decanos y directores que cualquier iniciativa o propuesta de cambio que deseen plantear a instancias superiores sea previamente discutida y consensuada entre los homólogos de ambos campus.

Todo lo anterior nos ha llevado a una gran conclusión que, pretendemos, afecte intensamente al trabajo que hagamos en adelante desde el CDC: Desde el punto de vista de la calidad, eficiencia y eficacia de los proyectos, el enfoque que le estamos dando a estos procesos supone una clara relativización de recursos y tiempos, pues cada iniciativa debe ser creada y evaluada al mismo tiempo desde tres ópticas diferentes: desde sus metas específicas, en términos de valor agregado para facilitar otras acciones y en función del remanente facilitador obtenido de experiencias previas o simultáneas.

Sin duda, para que una estrategia como esta funcione, es necesario transcender una visión cuantitativa y tangible de los procesos institucionales y migrar a otra en la que también se valoren las ganancias intangibles y no directamente cuantificables de los mismos; dado que son ellas las que redundan claramente en la calidad y consistencia del desarrollo institucional.

Las prisas y la impaciencia son los grandes demonios a los que se enfrentan los procesos de este tipo en las instituciones educativas pues, del mismo modo que los pasos "lentos" y firmes pueden convertirse en certeras catapultas hacia un incremento exponencial del desarrollo, se hace sumamente difícil mantener en el tiempo la confianza en que ello suceda y no dejarse llevar por la lógica de la inmediatez en este contexto precipitado y competitivo en el que nos encontramos.

Dejarse llevar por la inmediatez dificulta el desarrollo de procesos propios de las instituciones inteligentes, las instituciones que aprenden, se dan el tiempo para aprovechar en su beneficio el valor pedagógico del error y son capaces de verse proyectadas en un horizonte.

\section{Referencias}

Bain, K. (2005). Lo que hacen los mejores profesores universitarios. Valencia. Publicaciones de la Universidad de Valencia.

Di Napoli, R. (2010). Identidades académicas y gestión: ¿Una misión imposible?”. En Joan Rué y Laura Lodeiro (edit.), Equipos docentes y nuevas identidades académicas (pp. 189-201). Madrid: Narcea.

Lodeiro, L. (2013). Elicitación y representación del conocimiento experto de profesores universitarios: la construcción de redes y comunidades de aprendizaje como componente y condición de las "buenas prácticas docentes". (Tesis Doctoral inédita). Departamento de Didáctica y Organización Escolar. Universidad de Santiago de Compostela.

PUCMM (2011). Modelo Educativo. República Dominicana: Impresora Editora Teófilo.

Puentes, Y. (2006). Organizaciones escolares inteligentes. Bogotá: Cooperativa Editorial

Magisterio.

Rué, J. (2007). Enseñar en la Universidad: El EEES como reto para la Educación Superior. Madrid: Narcea.

Senge, P. (2005). La quinta disciplina: cómo construir una organización inteligente ( $3^{\mathrm{a}}$ ed.). Buenos Aires: Granica.

Universidad de Deusto (2001) Marco Pedagógico UD. Orientaciones Generales. Bilbao. Artes Gráficas Rontegui, S.A.L. 